\title{
Cost-Effectiveness of Interventions to Improve Maternal, Newborn and Child Health Outcomes: A WHO-CHOICE Analysis for Eastern Sub-Saharan Africa and South-East Asia
}

\author{
Karin Stenberg $^{{ }^{*} \oplus}$, Rory Watts ${ }^{2}$, Melanie Y. Bertram ${ }^{1}$, Kaia Engesveen ${ }^{3}$, Blerta Maliqi ${ }^{4}$, Lale Say ${ }^{5}$, Raymond \\ Hutubessy ${ }^{6}$
}

\begin{abstract}
Background: Information on cost-effectiveness allows policy-makers to evaluate if they are using currently available resources effectively and efficiently. Our objective is to examine the cost-effectiveness of health interventions to improve maternal, newborn and child health $(\mathrm{MNCH})$ outcomes, to provide global evidence relative to the context of two geographic regions.

Methods: We consider interventions across the life course from adolescence to pregnancy and for children up to 5 years old. Interventions included are those that fall within the areas of immunization, child healthcare, nutrition, reproductive health, and maternal/newborn health, and for which it is possible to model impact on MNCH mortality outcomes using the Lives Saved Tool (LiST). Generalized cost-effectiveness analysis (GCEA) was used to derive average cost-effectiveness ratios (ACERs) for individual interventions and combinations (packages). Costs were assessed from the health system perspective and reported in international dollars. Health outcomes were estimated and reported as the gain in healthy life years (HLYs) due to the specific intervention or combination. The model was run for 2 regions: Eastern sub-Saharan Africa (SSA-E) and South-East Asia (SEA).

Results: The World Health Organization (WHO) recommended interventions to improve MNCH are generally considered cost-effective, with the majority of interventions demonstrating ACERs below I\$100/HLY saved in the chosen settings (lowand middle-income countries [LMICs]). Best performing interventions are consistent across the two regions, and include family planning, neonatal resuscitation, management of pneumonia and neonatal infection, vitamin A supplementation, and measles vaccine. ACERs below I $\$ 100$ can be found across all delivery platforms, from community to hospital level. The combination of interventions into packages (such as antenatal care) produces favorable ACERs.

Conclusion: Within each region there are interventions which represent very good value for money. There are opportunities to gear investments towards high-impact interventions and packages for $\mathrm{MNCH}$ outcomes. Cost-effectiveness tools can be used at national level to inform investment cases and overall priority setting processes.

Keywords: Cost-Effectiveness, Maternal Health, Child health, Sub-Saharan Africa, South-East Asia

Copyright: $\odot 2021$ The Author(s); Published by Kerman University of Medical Sciences. This is an open-access article distributed under the terms of the Creative Commons Attribution License (https://creativecommons.org/licenses/ by/4.0), which permits unrestricted use, distribution, and reproduction in any medium, provided the original work is properly cited.

Citation: Stenberg K, Watts R, Bertram MY, Engesveen K, Maliqi B, Say L, Hutubessy R. Cost-effectiveness of interventions to improve maternal, newborn and child health outcomes: a WHO-CHOICE analysis for Eastern sub-Saharan Africa and South-East Asia. Int J Health Policy Manag. 2021;10(11):706-723. doi:10.34172/ijhpm.2021.07
\end{abstract}

\section{Article History:}

Received: 18 March 2020 Accepted: 31 January 2021 ePublished: 17 March 2021

View Video Summary

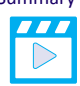

*Correspondence to:

Karin Stenberg

Email: stenbergk@who.int

\section{Introduction}

In 2017 an estimated 295000 women died from pregnancy or childbirth-related complications, and 5.3 million children under 5 years of age died in 2018. ${ }^{1}$ Deaths are inequitably distributed across the globe - More than half (3.3 million) of all these deaths happened in sub-Saharan Africa (SSA) followed by Central and Southern Asia with 28\% (1.8 million). ${ }^{1}$

Most of these deaths are preventable and can be avoided with the right investments. Following the adoption of the Millennium Development Goals (MDGs) in 2000, significant progress was made on goal 4 to reduce child mortality by two thirds, and goal 5 to reduce maternal mortality by three quarters. Recent reports indicate that maternal deaths decreased by 35\% between 2000 and 2017 and deaths of children under-five dropped by 59\% between 1990 and 2018 (1). Progress was also made on MDG 1 for nutrition: between 1990 and 2015, the global prevalence of underweight among children aged less than 5 declined from $25 \%$ to $14 \%$, nearly reaching the target of a $50 \%$ reduction. $^{2}$

These achievements represented significant improvements in population health, and were supported by increased coordinated funding from the development community. However, the goals were not universally achieved, and momentum needs to be maintained in order to address the unfinished agenda. The Sustainable Development Goals (SDGs) have set global targets for further reductions in 


\section{Key Messages}

Implications for policy makers

- Policy-makers in most countries consider cost-effectiveness to be an important criterion when making decisions around what health services to provide. The literature on cost-effectiveness of interventions to improve $\mathrm{MNCH}$ outcomes is rich and growing. There are many known highimpact interventions that have been recommended for a long time. However, service coverage remains limited for many interventions and the evidence base needs to be restated to support the case for investment.

- This paper provides an updated set of cost-effectiveness data for interventions that address $\mathrm{MNCH}$ outcomes for 2 geographic regions, following the methods of the WHO-CHOICE approach. These estimates provide a reference point for policy-makers to guide discussions around what interventions to include in national service packages to advance universal health coverage and attain the SDGs.

- Service packages will differ across settings based upon epidemiological profile, health budgets and local values. These global models form a starting point for the production of country-specific data to guide local discussions.

Implications for the public

Suboptimal maternal, newborn and child health $(\mathrm{MNCH})$ outcomes remains a major cause of burden of disease across low-and middle-income countries (LMICs). Many highly cost-effective interventions are not included in current benefit packages provided to the population, or have modest uptake among the population, either because of supply barriers (limited system capacity, low prioritization) or demand barriers (financial barriers, low demand). Estimates on cost-effectiveness can contribute to evidence-based discussions around what to provide in benefit packages. This can ultimately lead to greater investments in interventions that improve $\mathrm{MNCH}$, allowing populations in LMICs to benefit from better health outcomes.

maternal and child mortality, as well as retaining goals on ensuring universal access to sexual and reproductive healthcare. ${ }^{3}$ Within this agenda, good nutrition plays a key role: maternal and child undernutrition is estimated to contribute to 45 percent of deaths in children under five, ${ }^{4}$ and dietary iron deficiency is the fifth leading cause of disability adjusted life years among women of reproductive age. ${ }^{5}$

There are many high-impact interventions to improve maternal, newborn and child health $(\mathrm{MNCH})$ outcomes, for which evidence on effectiveness is well-known. ${ }^{6}$ Still, service uptake remains low across many settings. ${ }^{7}$ Many countries need to invest more and invest smarter. Evidence on cost-effectiveness allows policy-makers to evaluate if they are using currently available resources effectively and efficiently, and how they can best invest to achieve health targets and universal health coverage with limited resources. Whilst $\mathrm{MNCH}$ is generally proclaimed a priority area for investment across settings, actual budget allocation may not be sufficient to meet national targets. There is an increasing call for low- and middle-income countries (LMICs) to provide "investment cases" to indicate the value for money of proposed investments, for example in the area of noncommunicable diseases. ${ }^{8}$ This applies equally to $\mathrm{MNCH}$, for which the multi-partner Global Financing Facility (GFF) supports the development of investment cases in low-income settings. The GFF country investment cases aim to identify priority interventions to improve the health and nutrition of women, adolescents, and children. ${ }^{9}$ Evidence on locally contextualized cost-effectiveness data can help identify priorities. With a successful strategy, countries can access new financing from the World Bank, and can also be better informed for where to invest existing domestic resources.

As part of the World Health Organization's (WHO's) efforts to support Member States in the development of evidenceinformed health strategies, estimates on cost-effectiveness of prevention and treatment interventions are generated using standardized methods. ${ }^{10}$ The analysis presented here is part of an update of the WHO-Choosing Interventions that are Cost-Effective (CHOICE) programme of global level work.
In addition to the production of global level estimates, the CHOICE platform provides country contextualization tools to enable decision-makers to undertake their own analyses.

The CHOICE approach to cost-effectiveness is unique in three ways. Firstly, generalized cost-effectiveness is used. This is different to incremental cost-effectiveness which considers the value of adding new interventions at the margin of the existing package. The generalized cost-effectiveness analysis (GCEA) approach on the other hand, allows analysts to compare interventions compared to a "null" scenario, without considering the historical investments made. This allows the analysis to also take a critical view of the current package of available interventions, which may not always present the greatest value for money (for more details on the GCEA approach see methods paper in this series).${ }^{10}$ Secondly, a broad set of currently recommended interventions with adequate evidence are included in the analysis, initially individually and then as packages of care. The analysis applies a common methodology and assumptions across different disease areas, enabling interventions for different diseases to be compared fairly. Thus, here we analyze interventions to improve $\mathrm{MNCH}$ outcomes whereas other papers consider other intervention areas, ${ }^{11,12}$ and a separate summary paper considers the overall implications when a range of interventions are combined. ${ }^{13}$ Thirdly, a user-friendly tool kit is available for analysts to input local data and assumptions, to generate their own estimates.

The previous round of WHO-CHOICE cost-effectiveness analysis for $\mathrm{MNCH}$ was published in 2005. ${ }^{14,15}$ Among the highly cost-effective interventions identified were antenatal care for pregnant women, breastfeeding support, communitybased newborn care, and micronutrient supplementation for children.

The current study represents the first thorough re-analysis of the cost-effectiveness of interventions targeting $\mathrm{MNCH}$ outcomes by WHO since this time. The CHOICE methods and analysis platform have been updated and new health impact models developed. WHO Practice Guidelines have been updated in several areas (eg, antenatal care, ${ }^{16}$ intrapartum care, care for small and sick newborn, etc ${ }^{17}$ ). A broader set 
of interventions is considered in the new analysis, including nutritional supplementation before and during pregnancy; and an expanded set of vaccines. Furthermore, a userfriendly country contextualization tool has been developed, to accompany the global level analyses.

\section{Methods}

We examined the costs and impact on health of interventions to improve $\mathrm{MNCH}$ outcomes in 2 regions: Eastern subSaharan Africa (SSA-E) and South-East Asia (SEA). The regions are consistent with previous published analyses. ${ }^{14,15}$ For a full account of the methods used in this update of the WHO-CHOICE project, we refer to a separate paper that is published as part of this series. ${ }^{10}$ In this paper we describe specific methodology related to updating the analytical work for interventions targeting MNCH outcomes, including brief overviews of the models and the intervention assumptions used. The analysis used epidemiological and cost data for 2010, for the SSA-E and SEA Global Burden of Disease regions. Countries included in these regions are listed in Table 1.

\section{Interventions}

We considered interventions across the life course from adolescence to pregnancy and child birth, and during years 0-4 of the child. Interventions included immunization, child healthcare, nutrition, reproductive health, and maternal/ newborn healthcare that impact on mortality outcomes for pregnant women or women who recently delivered and children 0-4 years - including stillbirths. Interventions were included based on WHO recommendations, and for which an impact model existed to facilitate modelling. The analysis was undertaken using the Spectrum suite of impact models, and

Table 1. Countries Included in the Analysis

\begin{tabular}{lc}
\hline SEA & SSA-E \\
\hline Cambodia & Burundi \\
Indonesia & Comoros \\
Laos & Djibouti \\
Malaysia & Eritrea \\
Maldives & Ethiopia \\
Myanmar & Kenya \\
Philippines & Madagascar \\
Sri Lanka & Malawi \\
Thailand & Mauritius \\
Timor-Leste & Mozambique \\
Viet Nam & Rwanda \\
& Somalia \\
\hline & South Sudan \\
& Sudan \\
& Uganda \\
& Tanzania \\
\hline
\end{tabular}

Abbreviations: SEA, South East Asia; SSA-E, Eastern sub-Saharan Africa. is therefore limited to interventions included in Spectrum, in particular the Lives Saved Tool (LiST) ${ }^{18}$ and the Family Planning tool (FamPlan). ${ }^{19}$

We evaluated 37 interventions and 12 packages of combined interventions. These included interventions that allow individuals to exercise rights around deciding their family size (access to contraception, safe abortion); interventions which promote healthy practices and behaviors (eg, breastfeeding); interventions which prevent illness (eg, through immunization); and interventions which manage complications and illness (eg, complications arising at birth or infectious disease in childhood). Table 2 lists interventions with a description including the period of implementation/ life course (target population), the health programme, and service delivery platform.

It should be noted that some relevant interventions for maternal and child health, such as HPV vaccine, malaria and HIV/AIDS testing and treatment, were considered as part of analysis for other programmatic areas within the WHO-CHOICE series update and are therefore presented and discussed in other papers belonging to this series. ${ }^{11,12} \mathrm{An}$ exception is intermittent preventive treatment in pregnancy for malaria which we consider here as part of the antenatal care package and thus fit for inclusion. In addition to single interventions, we evaluate 12 packages that follow policyrelevant intervention combinations.

Interventions and packages are evaluated at three coverage levels, 50\%, 80\% and 95\%. Coverage targets for family planning cannot follow the same logic as they do not refer to a health need but a need for regulating pregnancy (which would never reach $100 \%$ ). The model therefore incorporates a calculation factor for contraceptive use. We apply a factor of 0.72 , which was derived by studying current contraceptive prevalence rates in the Organisation for Economic Co-operation and Development (OECD) countries, which according to recent data reach around $71 \%-72 \%$ for any method. ${ }^{20}$ Thus, a $50 \%$ coverage for family planning is run in the model as $50 \% \mathrm{x}$ $0.72=36 \%$.

\section{Health Outcomes}

Health outcomes were assessed using the Spectrum suite of impact models.

The LiST and FamPlan tools have been described in detail elsewhere. ${ }^{17,18}$ The Spectrum platform translates an increase in service coverage into effects on demography and health outcomes (eg, birth spacing, cause-specific mortality, nutritional status). ${ }^{21}$

For each intervention/package, the model generates information about the number of deaths that would have occurred in a scenario with zero coverage for the interventions(s) of interest: the "null" scenario. This is compared to a "scaleup" scenario where there is instantaneous scale-up from zero coverage in year 1 to the target coverage $(50 \%, 80 \%$ or $95 \%)$ in year 2, with target coverage then maintained for 100 years. To generate the "null" for maternal and child interventions, the SPECTRUM software cost-effectiveness tool runs the LiST and FamPlan modules accordingly, generating a scenario where coverage is zero for relevant interventions and the burden of 
Women of reproductive age (15-49 years) in union are provided with counseling and information on different methods for contraception, as well as the commodities required. This includes both traditional and modern methods such as pills and condoms, injectables, IUD, implant, and sterilization, based on countryspecific profile of contraceptive methods use.

Women of reproductive$$
\text { union }
$$

Maternal mortality

Maternal and newborn

Primary level (ific profile of contraceptive methods use.

Folic acid supplementation $\quad$ MNCH_2. FAS gestation, should take a folic acid supplement ( $400 \mu \mathrm{g}$ folic acid daily).

Safe abortion provided to women seeking to terminate pregnancy. Methods include manual or electric vacuum aspiration and medical abortion (mifepristone followed by a prostaglandin)

Treatment of women experiencing complications after undergoing unsafe abortions. Complications include haemorrhage, sepsis, peritonitis, and trauma to the cervix, vagina, uterus, and abdominal organs.

management

MNCH_4. PAC

Calcium supplementation in pregnant women for the prevention and management of pre-eclampsia/eclampsia

In populations with low dietary calcium intake, daily calcium supplementation (1.5 $\mathrm{g}-2.0 \mathrm{~g}$ oral elemental calcium) is recommended for pregnant women to reduce the risk of pre-eclampsia.

Daily oral iron and folic acid supplementation with $30 \mathrm{mg}$ to $60 \mathrm{mg}$ of elemental

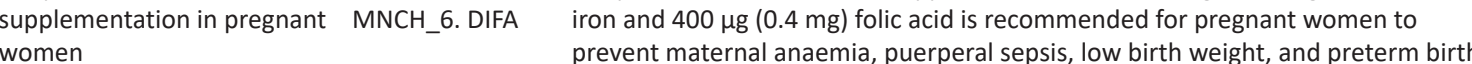
$\begin{aligned} & \text { supplementation in pregnant } \quad \mathrm{MNCH}_{-} 6 \text {. DIFA } \\ & \text { women }\end{aligned} \quad \begin{aligned} & \text { iron and } 400 \mu \mathrm{g}(0.4 \mathrm{mg}) \text { folic acid is recommended for pregnant women to } \\ & \text { prevent maternal anaemia, puerperal sepsis, low birth weight, and preterm birth. }\end{aligned}$ women

In undernourished populations, balanced energy and protein dietary

Balanced energy-protein

supplementation to pregnant

women living in areas with

MNCH_7. BEPS

supplementation is recommended for pregnant women to reduce the risk of stillbirths and small for gestational age neonates.

Tetanus toxoid vaccination

MNCH_8.TT

Two injections of tetanus toxoid vaccine.

Intermittent presumptive treatment of malaria in pregnancy

Intermittent presumptive treatment of malaria of all pregnant women living in areas endemic for Plasmodium falciparum.

Syphilis detection and treatment in pregnancy
Screening pregnant women by rapid plasma reagent test and treatment of seropositive cases with penicillin
Pregnant women

Newborn (0-1

Nutrition

Primary level

Women seeking to

terminate pregnancy

(incidence of abortion)

Women seeking to

terminate pregnancy

(incidence of abortion)

(aternat and

community

Maternal and Primary level

newborn

care

Maternal and

newborn

Hospital

Pregnant women

Maternal mortality and newborn

Primary level care

\section{Pregnant women} month)

Pregnant women living in areas with high food insecurity (based on income per capita)

\section{Newborn (0-1} month)

Pregnant women

Ma

Maternal mortality;

Newborn (0-1 Maternal and month)

newborn

Primary leve

Primary level

care, including community and outreach

Pregnant women living in areas endemic for Plasmodium falciparum

Maternal mortality; Malaria; Maternal Primary level stillbirths and newborn

Pregnant women

Maternal and

Primary level
Maternal mortality

$\begin{array}{ccc}\text { Newborn (0-1 } & \text { Maternal and } \\ \text { month); stillbirths } & \text { newborn } & \begin{array}{c}\text { Primary le } \\ \text { care }\end{array}\end{array}$




\begin{tabular}{|c|c|c|c|c|c|c|c|}
\hline Number & Intervention Name & Short Name & Intervention Definition & $\begin{array}{l}\text { Target Population in } \\
\text { Need of Intervention }\end{array}$ & Target for Impact & Health Programme & $\begin{array}{l}\text { Service Delivery } \\
\text { Platform }\end{array}$ \\
\hline 11 & $\begin{array}{l}\text { Hypertensive disease case } \\
\text { management in pregnancy }\end{array}$ & $\begin{array}{l}\text { MNCH_11. } \\
\text { CMHD }\end{array}$ & Management of moderate to severe hypertension without proteinuria. & Pregnant women & Maternal mortality & $\begin{array}{l}\text { Maternal and } \\
\text { newborn }\end{array}$ & $\begin{array}{l}\text { Primary level } \\
\text { care }\end{array}$ \\
\hline 12 & $\begin{array}{l}\text { Management of pre- } \\
\text { eclampsia (mild and severe) }\end{array}$ & MNCH_12. MPE & $\begin{array}{l}\text { Management of hypertension and mild pre-eclampsia through outpatient care; } \\
\text { management of severe pre-eclampsia through with magnesium sulfate through } \\
\text { inpatient care. }\end{array}$ & Pregnant women & Maternal mortality & $\begin{array}{l}\text { Maternal and } \\
\text { newborn }\end{array}$ & $\begin{array}{l}\text { Primary level } \\
\quad \text { care }\end{array}$ \\
\hline 13 & $\begin{array}{l}\text { Ectopic pregnancy case } \\
\text { management }\end{array}$ & MNCH_13.ECT & $\begin{array}{l}\text { Surgical intervention (laparoscopy or laparotomy) to interrupt an ectopic } \\
\text { pregnancy. }\end{array}$ & $\begin{array}{l}\text { Pregnant women with } \\
\text { ectopic pregnancy }\end{array}$ & Maternal mortality & $\begin{array}{l}\text { Maternal and } \\
\text { newborn }\end{array}$ & Hospital \\
\hline 14 & Neonatal resuscitation & MNCH_14. NR & $\begin{array}{l}\text { Detection of breathing problems and resuscitation of newborn when required, } \\
\text { using bag and mask. }\end{array}$ & Newborn & $\begin{array}{l}\text { Newborn } \\
\text { (0-1 month) }\end{array}$ & $\begin{array}{l}\text { Maternal and } \\
\text { newborn }\end{array}$ & $\begin{array}{l}\text { Primary level } \\
\quad \text { care }\end{array}$ \\
\hline 15 & $\begin{array}{l}\text { Clean cord care (clean birth } \\
\text { practices) }\end{array}$ & MNCH_15. CCC & Umbilical cord cleansing, with chlorhexidine or other disinfectant. & Newborn & $\begin{array}{l}\text { Newborn } \\
\text { (0-1 month) }\end{array}$ & $\begin{array}{l}\text { Maternal and } \\
\text { newborn }\end{array}$ & $\begin{array}{l}\text { Primary level } \\
\quad \text { care }\end{array}$ \\
\hline 16 & $\begin{array}{l}\text { Antibiotics for preterm } \\
\text { premature rupture of } \\
\text { membranes }\end{array}$ & $\begin{array}{l}\text { MNCH_16. } \\
\text { PPROM }\end{array}$ & $\begin{array}{l}\text { Hospitalization prior to delivery, with administration of oral antibiotics to women } \\
\text { with preterm premature rupture of membranes. }\end{array}$ & Pregnant women & Maternal mortality & $\begin{array}{l}\text { Maternal and } \\
\text { newborn }\end{array}$ & Hospital \\
\hline 17 & $\begin{array}{l}\text { Management of eclampsia } \\
\text { with magnesium-sulphate }\end{array}$ & $\begin{array}{l}\text { MNCH_17. } \\
\text { MEMS }\end{array}$ & $\begin{array}{l}\text { Management of convulsions associated with eclampsia, occurring ante-, intra- or } \\
\text { postpartum. }\end{array}$ & Pregnant women & Maternal mortality & $\begin{array}{l}\text { Maternal and } \\
\text { newborn }\end{array}$ & Hospital \\
\hline 18 & $\begin{array}{l}\text { Management of maternal } \\
\text { sepsis }\end{array}$ & MNCH_18. MMS & Management of sepsis symptoms within 42 days of delivery. & Pregnant women & Maternal mortality & $\begin{array}{l}\text { Maternal and } \\
\text { newborn }\end{array}$ & Hospital \\
\hline 19 & Promotion of breastfeeding & MNCH_19. BF & $\begin{array}{l}\text { Promotion of early and exclusive breastfeeding through skilled care providers and } \\
\text { community health workers. }\end{array}$ & Newborn & $\begin{array}{l}\text { Newborn (0-1 } \\
\text { month) and child } \\
\text { (1-59 months) }\end{array}$ & $\begin{array}{l}\text { Nutrition; Maternal } \\
\text { and newborn }\end{array}$ & $\begin{array}{l}\text { Primary level } \\
\text { care, including } \\
\text { community }\end{array}$ \\
\hline 20 & $\begin{array}{l}\text { Home visits for clean } \\
\text { postnatal practices }\end{array}$ & MNCH_20.CPNP & $\begin{array}{l}\text { Home visits within } 48 \text { hours of delivery to promote clean practices, specifically that } \\
\text { the mother washes her hands frequently, the child lives in a clean environment, } \\
\text { and no harmful practices are performed. }\end{array}$ & Newborn & $\begin{array}{l}\text { Newborn } \\
\text { (0-1 month) }\end{array}$ & $\begin{array}{l}\text { Maternal and } \\
\text { newborn }\end{array}$ & Community leve \\
\hline 21 & $\begin{array}{l}\text { Vitamin A supplementation } \\
\text { (0-4 years) }\end{array}$ & MNCH_21.VAS & $\begin{array}{l}\text { Vitamin A supplementation for children 6-59 months of age in countries (or } \\
\text { sub-national areas in some cases) where vitamin A deficiency is a public health } \\
\text { problem. }\end{array}$ & Children 6-59 months & $\begin{array}{l}\text { Child (1-59 } \\
\text { months) }\end{array}$ & Nutrition; Child & Community leve \\
\hline 22 & $\begin{array}{l}\text { Promotion of complementary } \\
\text { feeding }\end{array}$ & MNCH_22. CF & $\begin{array}{l}\text { Comprehensive counselling for the caregiver of a child (two full sessions) on } \\
\text { the importance of continued breastfeeding after } 6 \text { months of age along with } \\
\text { information on appropriate complementary feeding practices, through skilled care } \\
\text { providers and community health workers. }\end{array}$ & Children 6-11 months & $\begin{array}{c}\text { Child } \\
\text { (1-59 months) }\end{array}$ & Nutrition; Child & $\begin{array}{l}\text { Primary level } \\
\text { care, including } \\
\text { community }\end{array}$ \\
\hline 23 & DPT vaccine & MNCH_23. DPT & 3 doses of DPT vaccine. & Newborn & $\begin{array}{c}\text { Child (1-59 } \\
\text { months) }\end{array}$ & Immunization & $\begin{array}{l}\text { Primary level } \\
\text { care }\end{array}$ \\
\hline
\end{tabular}

710 International Journal of Health Policy and Management, 2021, 10(11), 706-723 
Table 2. Continued

\begin{tabular}{|c|c|c|c|c|c|c|c|}
\hline Number & Intervention Name & Short Name & Intervention Definition & $\begin{array}{l}\text { Target Population in } \\
\text { Need of Intervention }\end{array}$ & Target for Impact & Health Programme & $\begin{array}{l}\text { Service Delivery } \\
\text { Platform }\end{array}$ \\
\hline 24 & Hib vaccine & MNCH_24. HIB & 3 doses of $\mathrm{HiB}$ vaccine. & Newborn & $\begin{array}{l}\text { Child (1-59 } \\
\text { months) }\end{array}$ & Immunization & $\begin{array}{l}\text { Primary level } \\
\text { care }\end{array}$ \\
\hline 26 & Rotavirus vaccine & MNCH_26. ROTA & 3 doses of rotavirus vaccine. & Newborn & $\begin{array}{c}\text { Child } \\
\text { (1-59 months) }\end{array}$ & Immunization & $\begin{array}{l}\text { Primary level } \\
\text { care }\end{array}$ \\
\hline 28 & Measles vaccine & MNCH_28. MCV & 2 doses of measles vaccine. & Newborn & $\begin{array}{c}\text { Child } \\
\text { (1-59 months) }\end{array}$ & Immunization & $\begin{array}{l}\text { Primary level } \\
\text { care }\end{array}$ \\
\hline 29 & Kangaroo mother care & MNCH_29. KMC & $\begin{array}{l}\text { Inpatient support to KMC, defined as continuous skin-to-skin contact between a } \\
\text { mother and her newborn as well as frequent and exclusive breastfeeding. }\end{array}$ & Newborn & $\begin{array}{l}\text { Newborn } \\
\text { (0-1 month) }\end{array}$ & $\begin{array}{l}\text { Maternal and } \\
\text { newborn }\end{array}$ & Hospital \\
\hline 30 & $\begin{array}{l}\text { Full supportive care for } \\
\text { premature babies }\end{array}$ & MNCH_30. FSC & $\begin{array}{l}\text { Prematurely born neonates receive hospital-based full supportive care, including } \\
\text { KMC, feeding support/IV fluids, infection prevention/management, oxygen } \\
\text { provision, management of neonatal jaundice, nasal CPAP/IPPV (as required), and } \\
\text { surfactant for respiratory distress syndrome. }\end{array}$ & Newborn & $\begin{array}{l}\text { Newborn } \\
\text { (0-1 month) }\end{array}$ & $\begin{array}{l}\text { Maternal and } \\
\text { newborn }\end{array}$ & Hospital \\
\hline 31 & $\begin{array}{l}\text { Case management of severe } \\
\text { neonatal infection (sepsis/ } \\
\text { pneumonia) with full } \\
\text { supportive care }\end{array}$ & $\begin{array}{l}\text { MNCH_31. } \\
\text { CMSNI }\end{array}$ & $\begin{array}{l}\text { Case management of neonates with suspected sepsis/pneumonia treated with } \\
\text { hospital-based full supportive care, including oxygen, IV fluids, IV antibiotics, blood } \\
\text { transfusion, phototherapy, etc as needed, in addition to KMC. }\end{array}$ & Newborn & $\begin{array}{l}\text { Newborn } \\
\text { (0-1 month) }\end{array}$ & $\begin{array}{l}\text { Maternal and } \\
\text { newborn }\end{array}$ & Hospital \\
\hline 32 & $\begin{array}{l}\text { Facility-based management } \\
\text { of neonatal infection (sepsis/ } \\
\text { pneumonia) with injectable } \\
\text { (and oral) antibiotics }\end{array}$ & MNCH_32. CMNI & $\begin{array}{l}\text { Treatment of sepsis and infection at first level facility, with } 2 \text { days injectable } \\
\text { antibiotics followed by oral amoxicillin for } 7 \text { days. }\end{array}$ & Newborn & $\begin{array}{l}\text { Newborn } \\
\text { (0-1 month) }\end{array}$ & Child & $\begin{array}{l}\text { Primary level } \\
\text { care }\end{array}$ \\
\hline 33 & $\begin{array}{l}\text { Management of diarrhea } \\
\text { through oral rehydration } \\
\text { solution and zinc }\end{array}$ & $\begin{array}{l}\text { MNCH_33. } \\
\text { ORSzinc }\end{array}$ & Management of mild and moderate diarrhea with ORS and zinc tablets. & Children 0-59 months & $\begin{array}{c}\text { Child } \\
\text { (1-59 months) }\end{array}$ & Child & Community level \\
\hline 34 & $\begin{array}{l}\text { Community-based } \\
\text { management of pneumonia }\end{array}$ & $\begin{array}{l}\text { MNCH_34. } \\
\text { CCM_P }\end{array}$ & $\begin{array}{l}\text { Home visits for diagnosis and treatment of community-based management of } \\
\text { pneumonia in children below the age of } 5 \text { years, provided by community health } \\
\text { workers. }\end{array}$ & Children 0-59 months & $\begin{array}{l}\text { Child } \\
\text { (1-59 months) }\end{array}$ & Child & Community level \\
\hline
\end{tabular}




\begin{tabular}{|c|c|c|c|c|c|c|c|}
\hline Number & Intervention Name & Short Name & Intervention Definition & $\begin{array}{l}\text { Target Population in } \\
\text { Need of Intervention }\end{array}$ & Target for Impact & Health Programme & $\begin{array}{l}\text { Service Delivery } \\
\text { Platform }\end{array}$ \\
\hline 35 & $\begin{array}{l}\text { Antibiotics for treatment of } \\
\text { dysentery }\end{array}$ & MNCH_35. DYS & $\begin{array}{l}\text { Children with diarrhea presenting with blood in the stool receive a } 3 \text { day course of } \\
\text { ciprofloxacin and are re-evaluated after } 2 \text { days. }\end{array}$ & Children 0-59 months & $\begin{array}{l}\text { Child } \\
\text { (1-59 months) }\end{array}$ & Child & $\begin{array}{l}\text { Primary level } \\
\text { care }\end{array}$ \\
\hline 36 & $\begin{array}{l}\text { Facility-based management } \\
\text { of pneumonia }\end{array}$ & $\begin{array}{l}\text { MNCH_36. } \\
\text { FCM_P }\end{array}$ & Management of pneumonia with oral antibiotics. & Children 0-59 months & $\begin{array}{c}\text { Child } \\
\text { (1-59 months) }\end{array}$ & Child & $\begin{array}{l}\text { Primary level } \\
\text { care }\end{array}$ \\
\hline 37 & $\begin{array}{l}\text { Management of children with } \\
\text { severe acute malnutrition }\end{array}$ & $\begin{array}{l}\text { MNCH_37. } \\
\text { CMSAM }\end{array}$ & $\begin{array}{l}\text { Integrated management of children with severe acute malnutrition (<-3 Z-score) } \\
\text { through outpatient care for cases without medical complication }(80 \%) \text {, and } \\
\text { inpatient care for cases with medical complications and/or infants younger than } 6 \\
\text { months }(20 \%) \text {. }\end{array}$ & Children 0-59 months & $\begin{array}{l}\text { Child } \\
\text { (1-59 months) }\end{array}$ & Nutrition; Child & $\begin{array}{l}\text { Primary level } \\
\text { care, including } \\
\text { community }\end{array}$ \\
\hline \multicolumn{8}{|c|}{ Packages } \\
\hline P1 & $\begin{array}{l}\text { Preventing and managing } \\
\text { unplanned pregnancy }\end{array}$ & MNCH_P1. UPP & $\begin{array}{l}\text { Family planning counseling integrated into safe abortion and post-abortion care ( } 3 \\
\text { interventions: includes } \# 1, \# 3 \text {, and } \# 4) \text {. }\end{array}$ & $\begin{array}{l}\text { Women seeking to plan } \\
\text { pregnancy }\end{array}$ & Maternal mortality & $\begin{array}{l}\text { Maternal and } \\
\text { newborn }\end{array}$ & $\begin{array}{l}\text { Primary level } \\
\text { care; (hospital } \\
\text { level for post- } \\
\text { abortion care) }\end{array}$ \\
\hline P2 & $\begin{array}{l}\text { Comprehensive antenatal } \\
\text { care }\end{array}$ & MNCH_P2. ANC & $\begin{array}{l}\text { A package of antenatal care aligned with WHO guidelines and including tetanus } \\
\text { toxoid vaccine, iron supplementation, calcium supplementation, balanced energy } \\
\text { supplementation, syphilis detection and treatment, hypertensive disorder case } \\
\text { management, } \mathrm{MgSO}_{4} \text { management of pre-eclampsia, and IPTM where relevant (8 } \\
\text { interventions: includes \#5-12). }\end{array}$ & Pregnant women & $\begin{array}{l}\text { Maternal mortality; } \\
\text { Newborn (0-1 } \\
\text { month); Stillbirths }\end{array}$ & $\begin{array}{l}\text { Maternal and } \\
\text { newborn }\end{array}$ & $\begin{array}{l}\text { Primary level } \\
\quad \text { care }\end{array}$ \\
\hline P3 & $\begin{array}{l}\text { Skilled assistance for normal } \\
\text { delivery }\end{array}$ & MNCH_P3. SBA & $\begin{array}{l}\text { Skilled assistance with facility-based births, not necessarily EmOC level. } \\
\text { Components include immediate assessment and stimulation, support during } \\
\text { labor and delivery, active management of the third stage of labour, newborn } \\
\text { resuscitation, and clean cord care. ( } 5 \text { interventions, of which \#14 and \#15 listed } \\
\text { above as individual interventions). }\end{array}$ & Pregnant women & $\begin{array}{l}\text { Maternal mortality; } \\
\text { Newborn (0-1 } \\
\text { month); Stillbirths }\end{array}$ & $\begin{array}{l}\text { Maternal and } \\
\text { newborn }\end{array}$ & $\begin{array}{l}\text { Primary level } \\
\quad \text { care }\end{array}$ \\
\hline P4 & $\begin{array}{l}\text { Skilled assistance for normal } \\
\text { delivery + family planning }\end{array}$ & $\begin{array}{l}\text { MNCH_P4. SBA } \\
+\mathrm{FP}\end{array}$ & $\begin{array}{l}\text { P3+ integrated postpartum family planning advice and contraceptive provision ( } 6 \\
\text { interventions). }\end{array}$ & Pregnant women & $\begin{array}{l}\text { Maternal mortality; } \\
\text { Newborn (0-1 } \\
\text { month); Stillbirths }\end{array}$ & $\begin{array}{l}\text { Maternal and } \\
\text { newborn }\end{array}$ & $\begin{array}{l}\text { Primary level } \\
\quad \text { care }\end{array}$ \\
\hline P5 & $\begin{array}{l}\text { Skilled delivery + } \\
\text { management of } \\
\text { complications }\end{array}$ & $\begin{array}{l}\text { MNCH_P5. SBA + } \\
\text { comp }\end{array}$ & $\begin{array}{l}\text { Skilled assistance for normal deliveries with quick and efficient referral to quality } \\
\text { emergency obstetric care services when complications arise, }+ \text { induction of labor }+ \\
\text { full supportive care for newborn infections ( } 12 \text { interventions). }\end{array}$ & Pregnant women & $\begin{array}{l}\text { Maternal mortality; } \\
\text { Newborn (0-1 } \\
\text { month); Stillbirths }\end{array}$ & $\begin{array}{l}\text { Maternal and } \\
\text { newborn }\end{array}$ & $\begin{array}{l}\text { Primary level } \\
\text { care + hospital }\end{array}$ \\
\hline P6 & $\begin{array}{l}\text { Skilled delivery }+ \\
\text { management of } \\
\text { complications + family } \\
\text { planning }\end{array}$ & $\begin{array}{l}\text { MNCH_P6.SBA+ } \\
\text { comp + FP }\end{array}$ & $\begin{array}{l}\text { P5+ integrated postpartum family planning advice and contraceptive provision (13 } \\
\text { interventions of which most are listed above as individual interventions). }\end{array}$ & Pregnant women & $\begin{array}{l}\text { Maternal mortality; } \\
\text { Newborn (0-1 } \\
\text { month); Stillbirths }\end{array}$ & $\begin{array}{l}\text { Maternal and } \\
\text { newborn }\end{array}$ & $\begin{array}{l}\text { Primary level } \\
\text { care + hospital }\end{array}$ \\
\hline
\end{tabular}




\begin{tabular}{|c|c|c|c|c|c|c|c|}
\hline Number & Intervention Name & Short Name & Intervention Definition & $\begin{array}{l}\text { Target Population in } \\
\text { Need of Intervention }\end{array}$ & Target for Impact & Health Programme & $\begin{array}{l}\text { Service Delivery } \\
\text { Platform }\end{array}$ \\
\hline P7 & $\begin{array}{l}\text { Case management of } \\
\text { newborn complications at } \\
\text { referral level }\end{array}$ & MNCH_P7. CMNC & $\begin{array}{l}\text { Full supportive care for premature babies + Case management of severe neonatal } \\
\text { infection (sepsis/pneumonia) with full supportive care ( } 2 \text { interventions: combines } \\
\# 30 \text { and \# 31). }\end{array}$ & $\begin{array}{c}\text { Newborns with } \\
\text { complications } \\
\text { (prematurity, severe } \\
\text { infection) }\end{array}$ & $\begin{array}{l}\text { Newborn } \\
(0-1 \text { month) }\end{array}$ & $\begin{array}{l}\text { Maternal and } \\
\text { newborn }\end{array}$ & Hospital \\
\hline P8 & $\begin{array}{l}\text { Community-based newborn } \\
\text { and child care }\end{array}$ & $\begin{array}{l}\text { MNCH_P8. } \\
\text { CBNCC }\end{array}$ & $\begin{array}{l}\text { Community-based preventive and curative care (breastfeeding promotion, } \\
\text { postnatal visits, vitamin A supplementation, management of infections, } \\
\text { pneumonia and diarrhea), ( } 5 \text { interventions, listed above as \#19-21+\#33 + \#34). }\end{array}$ & $\begin{array}{l}\text { Newborns and children } \\
0-59 \text { months }\end{array}$ & $\begin{array}{l}\text { Newborn (0-1 } \\
\text { month) and child } \\
(1-59 \text { months })\end{array}$ & $\begin{array}{l}\text { Maternal and } \\
\text { newborn; Child }\end{array}$ & Community \\
\hline P9 & $\begin{array}{l}\text { Infant and young child } \\
\text { feeding }\end{array}$ & MNCH_P9. IYCF & $\begin{array}{l}\text { Breastfeeding promotion + Complementary feeding promotion + Vitamin A } \\
\text { supplementation ( } 3 \text { interventions, listed above as } \# 19, \# 21, \# 22) \text {. }\end{array}$ & $\begin{array}{l}\text { Newborns and children } \\
0-59 \text { months }\end{array}$ & $\begin{array}{l}\text { Child (1-59 } \\
\text { months) }\end{array}$ & Nutrition; Child & $\begin{array}{l}\text { Community and } \\
\text { primary level } \\
\text { care }\end{array}$ \\
\hline P10 & $\begin{array}{l}\text { Routine EPI (measles, } \\
\text { diphtheria, pertussis, } \\
\text { tetanus, and tuberculosis) }\end{array}$ & MNCH_P10. EPI & BCG, DTP, Hib, and measles immunization (4 interventions). & $\begin{array}{l}\text { Newborns and children } \\
0-59 \text { months }\end{array}$ & $\begin{array}{l}\text { Child (1-59 } \\
\text { months) }\end{array}$ & Immunization & $\begin{array}{l}\text { Primary level } \\
\text { care }\end{array}$ \\
\hline P11 & $\begin{array}{l}\text { Routine EPI + additional } \\
\text { vaccines }\end{array}$ & $\begin{array}{l}\text { MNCH_P11. } \\
\text { EPI+ROTA+PCV }\end{array}$ & BCG, DTP, Hib, measles, rotavirus and pneumococcal vaccines (6 interventions). & $\begin{array}{l}\text { Newborns and children } \\
0-59 \text { months }\end{array}$ & $\begin{array}{l}\text { Newborn (0-1 } \\
\text { month) and child } \\
\text { (1-59 months) }\end{array}$ & Immunization & $\begin{array}{l}\text { Primary level } \\
\text { care }\end{array}$ \\
\hline P12 & $\begin{array}{l}\text { Primary level integrated } \\
\text { management of the sick } \\
\text { child (includes link to the } \\
\text { community) }\end{array}$ & MNCH_P12. IMCl & $\begin{array}{l}\text { Management of diarrhea, dysentery, pneumonia, and severe malnutrition (4 } \\
\text { interventions - combines \#33, with \#35, \#36,\#37). }\end{array}$ & Children 0-59 months & $\begin{array}{l}\text { Child (1-59 } \\
\text { months) }\end{array}$ & Child & $\begin{array}{l}\text { Primary level } \\
\text { care }\end{array}$ \\
\hline
\end{tabular}

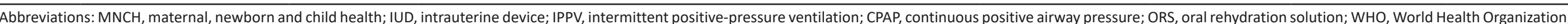

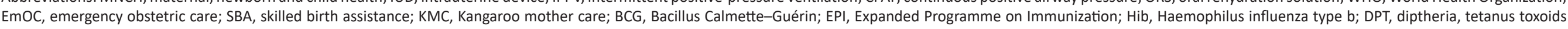
and pertussis. 
disease increases accordingly.

Results are analyzed by country and year. The model accounts for the synergies in effects and causes such that lives saved are not double counted. Deaths averted include maternal, newborn, child (0-4 years) and stillbirths. Deaths averted are converted into healthy life years (HLYs) gained based on age at time of death, average life expectancy for that age bracket, and the average health state valuation for a life saved from age at death until life expectancy. The model is largely restricted to impact measured in terms of mortality changes, however we did include both the "years of life lost" and (average) "years lived with disability" component for the future stream of life saved by the interventions. This allows us to compare the cost-effectiveness ratios in \$/HLY gained with those from other disease areas. Disease weights used in the HLY calculations are from the Global Burden of Disease study, 2010. ${ }^{22}$ The HLY estimations are thus based on DALY data, and the distinction between DALYs and HLYs is a distinction in name only, not in nature (we believe that "HLYs gained" is a more intuitive measure for decision-makers than "DALYs" when considering investments).

The analysis presented here is constrained by the evidence included within the LiST model, and is therefore largely restricted to evaluating impact on mortality. For interventions such as family planning that do not directly impact on mortality, the effect was measured in terms of averted maternal mortality resulting from fewer births. While many interventions are known to also reduce morbidity, unfortunately a lack of reliable data has prevented inclusion of such impact estimates within the LIST model, and therefore the benefits of some interventions are underestimated. For effect sizes used within the analysis see Supplementary file 1.

\section{Cost Assumptions}

Costing of interventions followed a standardized framework developed for WHO-CHOICE, and includes patient level delivery costs, programme costs and health system (service delivery) costs. Costs are estimated from the perspective of the government as the health system funder. Costs incurred by patients outside of the direct healthcare (eg, fees for transport) are not included in the analysis.

The GCEA analytical perspective assumes there is sufficient health system capacity in place to support the intervention. Quantity assumptions are based on adherence to WHO guidelines for the intervention of interest, and the analysis uses patient level intervention costs from the OneHealth Tool, ${ }^{23}$ with detailed prices for medicines and supplies, and with an additional 13\% markup rate applied to medicine and supply prices to cover logistics costs. ${ }^{24}$ Programme costs follow a standard methodology, ${ }^{24}$ with prices from the WHOCHOICE price database (https://www.who.int/choice) and capital expenses annuitized over the lifetime of the good. Health system (service delivery) costs use WHO-CHOICE country-specific estimates for inpatient and outpatient costs, ${ }^{25}$ combined with updated estimates for salary cost of specific health workers. ${ }^{26}$ The recent updates to the price databases used by WHO-CHOICE have overall higher cost predictions than previous database. ${ }^{24-26}$ All prices are presented here in
2010 International Dollars (2010 was chosen as the baseline year to align with the 2010 Global Burden of Disease study epidemiological data).

Table 2 provides information on assumptions used for target population and mode of delivery. The Supplementary file 2 provides additional detail on cost inputs - including average outpatient visits, health worker time, and health products, per intervention. Costs were estimated for each country using country-specific prices in 2010 I\$ and then combined into an aggregate cost for each region, then divided by the total population per sub-region, across 100 years.

\section{Comparing Interventions}

All interventions and packages were individually compared to the hypothetical "null" scenario in which the effects of all currently implemented interventions are removed. ${ }^{10}$ Health impacts and costs are thus calculated as the difference between the scale-up and null scenarios. All costs and impacts are assessed over a 100-year time frame from 20102110 , with year-by year results being generated. The average cost-effectiveness ratios (ACERs) were calculated by dividing the total cost for scale-up by the total health gain.

In the main scenario presented here, costs are discounted at $3 \%$ per annum, whereas HLYs are not discounted $(0 \%$ discount rate for impact). ${ }^{10}$ We also analyzed results when costs and HLYs are both discounted at 3\% (results in Supplementary file 3). Additional sensitivity analysis was performed through varying the coverage rates and applying one-way deterministic sensitivity analysis of $25 \%$ higher or lower costs for medicines and medical supplies.

Designing a package will require prioritization within a budget constraint. The marginal addition of interventions and packages is explored in order to describe an "expansion path" for an essential benefit package for $\mathrm{MNCH}$ impact. The expansion path describes the order in which interventions should be implemented in order to maximize health outcomes for any given budget, assuming that cost-effectiveness is the only criteria considered, and no system constraints. Here, we assess how an expansion path might be constructed in a hypothetical setting in South East Asia. For clarity, we include only interventions at $95 \%$ coverage, and apply a maximum budget of 4 million I\$. We adjusted impact and costs in cases where previous interventions on the expansion path already captured some of the expected health gains.

\section{Results}

ACERs for $95 \%$ coverage are presented in tables 3 and 4 . Costeffectiveness ratios decrease as coverage levels increase from $50 \%$ to $80 \%$ and $95 \%$ (see Supplementary file 3 for results), reflecting economies of scale built into the programme costs. ${ }^{24}$ In general, ACERs are much higher in the South East Asia region than in SSA-E. However, within each region there are interventions which represent very good value for money (Tables 3 and 4).

In SSA-E, 27 single interventions and all 12 packages have ACERs below I $\$ 100$, with 8 interventions between $\$ 100-\$ 400$ and 2 interventions above $\$ 1000$.

In SEA, half (26) of the interventions and packages have 
Table 3. Interventions Presented in Bands of Cost-Effectiveness, SSA-E (95\% Population Coverage, 3\% Discount Rate for Costs, $0 \%$ Discount Rate for Health Effects)

\begin{tabular}{|c|c|c|c|c|c|}
\hline Intervention & Short Name & ACER & $\begin{array}{l}\text { Cost Per } 1 \text { Million } \\
\text { Population (I\$) }\end{array}$ & $\begin{array}{l}\text { HLY Per } 1 \text { Million } \\
\text { Population }\end{array}$ & $\begin{array}{c}\text { Target Population } \\
\text { Group }\end{array}$ \\
\hline \multicolumn{6}{|l|}{$<\$ 10 /$ HLY gained } \\
\hline Skilled assistance for normal delivery + family planning & $\mathrm{MNCH} \_\mathrm{P} 4 . \mathrm{SBA}+\mathrm{FP}$ & 1.2 & 6266654 & 5192430 & Pregnant women \\
\hline Family planning & MNCH_1. FP & 2.7 & 14131612 & 5256634 & Pregnant women \\
\hline Skilled delivery + management of complications + family planning & MNCH_P6. SBA + comp + FP & 0.4 & 22857472 & 54115655 & Pregnant women \\
\hline Preventing and managing unplanned pregnancy & MNCH_P1. UPP & 0.7 & 1802557 & 2523029 & Pregnant women \\
\hline Neonatal resuscitation & MNCH_14. NR & 1.0 & 134391 & 131675 & Newborn \\
\hline Community-based management of pneumonia & MNCH_34.CCM_P & 2.5 & 154459 & 61116 & Child \\
\hline Facility-based management of pneumonia & MNCH_36. FCM_P & 3.5 & 210934 & 61116 & Child \\
\hline Case management of severe neonatal infection (sepsis/pneumonia) with full supportive care & MNCH_31. CMSNI & 3.6 & 149142 & 41339 & Newborn \\
\hline Vitamin A supplementation (0-4 years) & MNCH_21. VAS & 7.1 & 242300 & 34309 & Child \\
\hline Facility-based management of neonatal infection (sepsis/pneumonia) with injectable (and oral) antibiotics & MNCH_32. CMNI & 8.2 & 142418 & 17303 & Newborn \\
\hline \multicolumn{6}{|l|}{ Between $\$ 10$ and $<\$ 100 /$ HLY gained } \\
\hline Measles vaccine & MNCH_28. MCV & 10.1 & 200492 & 19891 & Child \\
\hline Home visits for clean postnatal practices & MNCH_20. CPNP & 11.5 & 215967 & 18699 & Newborn \\
\hline Infant and young child feeding & MNCH_P9. IYCF & 11.7 & 629808 & 53789 & Child \\
\hline Primary level integrated management of the sick child (includes link to the community) & MNCH_P12. IMCl & 12.3 & 1820209 & 147912 & Child \\
\hline Community-based newborn and child care & MNCH_P8. CBNCC & 13.8 & 2434145 & 176074 & Child \\
\hline Case management of newborn complications at referral level & MNCH_P7. CMNC & 14.4 & 979674 & 68096 & Newborn \\
\hline Routine EPI (measles, diphtheria, pertussis, tetanus, and tuberculosis) & MNCH_P10.EPI & 14.4 & 469958 & 32672 & Child \\
\hline Management of children with severe acute malnutrition & MNCH_37. CMSAM & 16.5 & 234943 & 14233 & Child \\
\hline H. influenzae b vaccine & MNCH_24. HIB & 17.5 & 650325 & 37210 & Child \\
\hline Kangaroo mother care & MNCH_29. KMC & 20.1 & 249627 & 12411 & Newborn \\
\hline Routine EPI + additional vaccines (rotavirus, pneumococcal, HepB - if we use the pentavalent ) & MNCH_P11. EPI + ROTA + PCV & 20.1 & 1023615 & 51010 & Child \\
\hline Pentavalent vaccine (DPT + Hep B + Hib) & MNCH_27. PENTA (DPT + HEPB + HIB) & 20.1 & 296640 & 14791 & Child \\
\hline Management of diarrhea through oral rehydration solution and zinc & MNCH_33. ORSzinc & 22.3 & 1818802 & 81557 & Child \\
\hline Tetanus toxoid vaccination & MNCH_8. TT & 22.6 & 227810 & 10073 & Pregnant women \\
\hline Clean cord care (clean birth practices) & MNCH_15. CCC & 23.8 & 137059 & 5759 & Newborn \\
\hline Syphilis detection and treatment in pregnancy & MNCH_10.SYP & 24.8 & 233088 & 9417 & Pregnant women \\
\hline
\end{tabular}




\begin{tabular}{|c|c|c|c|c|c|}
\hline Intervention & Short Name & ACER & $\begin{array}{l}\text { Cost Per } 1 \text { Million } \\
\text { Population (I\$) }\end{array}$ & $\begin{array}{l}\text { HLY Per } 1 \text { Million } \\
\text { Population }\end{array}$ & $\begin{array}{c}\text { Target Population } \\
\text { Group }\end{array}$ \\
\hline Comprehensive antenatal care & MNCH_P2. ANC & 26.8 & 1019342 & 37988 & Pregnant women \\
\hline Balanced energy-protein supplementation to pregnant women with insecure food availability & MNCH_7. BEPS & 27.9 & 427704 & 15336 & Pregnant women \\
\hline Promotion of breastfeeding & MNCH_19. BF & 29.0 & 331717 & 11449 & Newborn \\
\hline Skilled assistance for normal delivery & MNCH_P3.SBA & 29.6 & 4558206 & 153977 & Pregnant women \\
\hline Rotavirus vaccine & MNCH_26. ROTA & 30.1 & 386284 & 12840 & Child \\
\hline Pneumococcal vaccine & MNCH_25. PCV & 34.9 & 750344 & 21498 & Child \\
\hline Promotion of complementary feeding & MNCH_22. CF & 36.7 & 215932 & 5882 & Child \\
\hline Intermittent presumptive treatment of malaria & MNCH_9. IPTM & 53.7 & 201762 & 3755 & Pregnant women \\
\hline Skilled delivery + management of complications & MNCH_P5. SBA + comp & 56.9 & 12423164 & 218180 & Pregnant women \\
\hline Full supportive care for premature babies & MNCH_30. FSC & 62.7 & 726906 & 11593 & Newborn \\
\hline Management of pre-eclampsia (mild and severe) & MNCH_12.MPE & 85.4 & 146842 & 1720 & Pregnant women \\
\hline Management of maternal sepsis & MNCH_18. MMS & 93.0 & 203655 & 2190 & Pregnant women \\
\hline Hypertensive disease case management in pregnancy & MNCH_11. CMHD & 94.9 & 135050 & 1424 & Pregnant women \\
\hline \multicolumn{6}{|l|}{ Between $\$ 100$ and $<\$ 1000 /$ HLY gained } \\
\hline Daily iron and folic acid supplementation in pregnant women & MNCH_6. DIFA & 111.2 & 247292 & 2224 & Pregnant women \\
\hline DPT vaccine & MNCH_23. DPT & 111.9 & 432267 & 3862 & Child \\
\hline Antibiotics for treatment of dysentery & MNCH_35. DYS & 112.7 & 374913 & 3325 & Child \\
\hline Safe abortion services & MNCH_3. SA & 144.1 & 151490 & 1051 & Pregnant women \\
\hline Antibiotics for preterm premature rupture of membranes & MNCH_16. PPROM & 184.2 & 163204 & 886 & Pregnant women \\
\hline Post abortion case management & MNCH_4.PAC & 197.5 & 155505 & 787 & Pregnant women \\
\hline Management of eclampsia with magnesium-sulphate & MNCH_17. MEMS & 293.9 & 189484 & 645 & Pregnant women \\
\hline Folic acid supplementation & MNCH_2. FAS & 355.9 & 191051 & 537 & Pregnant women \\
\hline \multicolumn{6}{|l|}{ Between $\$ 1000$ and $<\$ 10000 / \mathrm{HLY}$ gained } \\
\hline Ectopic pregnancy case management & MNCH_13.ECT & 1156.2 & 160480 & 139 & Pregnant women \\
\hline Calcium supplementation in pregnant women for the prevention and management of pre-eclampsia/eclampsia & MNCH_5.CS & 1310.6 & 541387 & 413 & Pregnant women \\
\hline
\end{tabular}

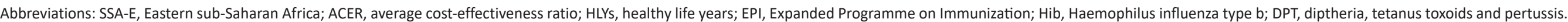


Table 4. Interventions Presented in Bands of Cost-Effectiveness, SEA (95\% Population Coverage, 3\% Discount Rate for Costs, 0\% Discount Rate for Health Effects)

\begin{tabular}{|c|c|c|c|c|c|}
\hline Intervention & Short Name & ACER & $\begin{array}{l}\text { Cost Per } 1 \text { Million } \\
\text { Population (I\$) }\end{array}$ & $\begin{array}{l}\text { HLY Per } 1 \text { Million } \\
\text { Population }\end{array}$ & $\begin{array}{c}\text { Target Population } \\
\text { Group }\end{array}$ \\
\hline \multicolumn{6}{|l|}{$<\$ 10 / \mathrm{HLY}$ gained } \\
\hline Skilled assistance for normal delivery + family planning & $\mathrm{MNCH} \_\mathrm{P} 4 . \mathrm{SBA}+\mathrm{FP}$ & 22.1 & 231276 & 3256415 & Pregnant women \\
\hline Neonatal resuscitation & MNCH_14.NR & 1.7 & 43712 & 25726 & Newborn \\
\hline Skilled delivery + management of complications + family planning & MNCH_P6. SBA + comp + FP & 35.7 & 242103 & 3356485 & Pregnant women \\
\hline Community-based management of pneumonia & MNCH_34.CCM_P & 5.0 & 49649 & 9890 & Child \\
\hline Case management of severe neonatal infection (sepsis/pneumonia) with full supportive care & MNCH_31. CMSNI & 6.6 & 65864 & 10035 & Newborn \\
\hline \multicolumn{6}{|l|}{ Between $\$ 10$ and $<\$ 100 / H L Y$ gained } \\
\hline Facility-based management of pneumonia & MNCH_36. FCM_P & 10.3 & 101727 & 9890 & Child \\
\hline Family planning & MNCH_1. FP & 11.2 & 2334143 & 207711 & Pregnant women \\
\hline Vitamin A supplementation (0-4 years) & MNCH_21.VAS & 13.3 & 97569 & 7349 & Child \\
\hline Measles vaccine & MNCH_28. MCV & 15.6 & 110365 & 7085 & Child \\
\hline Home visits for clean postnatal practices & MNCH_20. CPNP & 19.0 & 80671 & 4241 & Newborn \\
\hline Facility-based management of neonatal infection (sepsis/pneumonia) with injectable (and oral) antibiotics & MNCH_32. CMNI & 19.7 & 51321 & 2606 & Newborn \\
\hline Routine EPI (measles, diphtheria, pertussis, tetanus, and tuberculosis) & MNCH_P10.EPI & 30.6 & 298776 & 9755 & Child \\
\hline Routine EPI + additional vaccines (rotavirus, pneumococcal, Hep B - if we use the pentavalent) & MNCH_P11. EPI + ROTA + PCV & 38.9 & 491718 & 12636 & Child \\
\hline Community-based newborn and child care & MNCH_P8. CBNCC & 39.8 & 1026101 & 25773 & Child \\
\hline Kangaroo mother care & MNCH_29. KMC & 44.6 & 173481 & 3889 & Newborn \\
\hline Infant and young child feeding & MNCH_P9. IYCF & 47.5 & 498603 & 10501 & Child \\
\hline Clean cord care (clean birth practices) & MNCH_15. CCC & 49.2 & 43504 & 885 & Newborn \\
\hline Management of children with severe acute malnutrition & MNCH_37. CMSAM & 53.6 & 107652 & 2007 & Child \\
\hline Primary level integrated management of the sick child (includes link to the community) & MNCH_P12. IMCl & 54.2 & 905747 & 16697 & Child \\
\hline Promotion of breastfeeding & MNCH_19. BF & 54.6 & 135622 & 2482 & Newborn \\
\hline Management of diarrhea through oral rehydration solution and zinc & MNCH_33. ORSzinc & 63.3 & 575694 & 9102 & Child \\
\hline Case management of newborn complications at referral level & MNCH_P7.CMNC & 67.6 & 847152 & 12534 & Newborn \\
\hline Pentavalent vaccine (DPT + Hep B + Hib) & MNCH_27. PENTA (DPT + HEPB + HIB) & 74.3 & 167531 & 2254 & Child \\
\hline Balanced energy-protein supplementation to pregnant women with insecure food availability & MNCH_7. BEPS & 87.3 & 81532 & 934 & Pregnant women \\
\hline
\end{tabular}




\section{Table 4. Continued}

\begin{tabular}{|c|c|c|c|c|c|}
\hline Intervention & Short Name & ACER & $\begin{array}{l}\text { Cost Per } 1 \text { Million } \\
\text { Population (I\$S) }\end{array}$ & $\begin{array}{l}\text { HLY Per } 1 \text { Million } \\
\text { Population }\end{array}$ & $\begin{array}{c}\text { Target Population } \\
\text { Group }\end{array}$ \\
\hline H. influenzae b vaccine & MNCH_24. HIB & 90.0 & 429887 & 4778 & Child \\
\hline \multicolumn{6}{|l|}{ Between $\$ 100$ and $<\$ 1000 /$ HLY gained } \\
\hline Syphilis detection and treatment in pregnancy & MNCH_10.SYP & 102.7 & 158103 & 1539 & Pregnant women \\
\hline Promotion of complementary feeding & MNCH_22. CF & 109.9 & 122560 & 1115 & Child \\
\hline Comprehensive antenatal care & MNCH_P2. ANC & 125.9 & 705147 & 5600 & Pregnant women \\
\hline Skilled assistance for normal delivery & MNCH_P3.SBA & 128.6 & 3030550 & 23565 & Pregnant women \\
\hline Tetanus toxoid vaccination & MNCH_8. TT & 136.7 & 155763 & 1139 & Pregnant women \\
\hline Pneumococcal vaccine & MNCH_25.PCV & 138.1 & 468206 & 3391 & Child \\
\hline Intermittent presumptive treatment of malaria & MNCH_9. IPTM & 142.6 & 113448 & 795 & Pregnant women \\
\hline Rotavirus vaccine & MNCH_26. ROTA & 149.6 & 266987 & 1785 & Child \\
\hline Full supportive care for premature babies & MNCH_30. FSC & 154.9 & 561212 & 3624 & Newborn \\
\hline Skilled delivery + management of complications & MNCH_P5.SBA + comp & 199.7 & 6869115 & 34392 & Pregnant women \\
\hline Daily iron and folic acid supplementation in pregnant women & MNCH_6. DIFA & 236.1 & 166450 & 705 & Pregnant women \\
\hline Hypertensive disease case management in pregnancy & MNCH_11.CMHD & 307.4 & 42471 & 138 & Pregnant women \\
\hline Management of pre-eclampsia (mild and severe) & MNCH_12. MPE & 342.7 & 56815 & 166 & Pregnant women \\
\hline DPT vaccine & MNCH_23.DPT & 556.9 & 349890 & 628 & Child \\
\hline Antibiotics for treatment of dysentery & MNCH_35. DYS & 581.5 & 230594 & 397 & Child \\
\hline Management of eclampsia with magnesium-sulphate & MNCH_17. MEMS & 733.0 & 90613 & 124 & Pregnant women \\
\hline Safe abortion services & MNCH_3.SA & 854.5 & 68083 & 80 & Pregnant women \\
\hline Post abortion case management & MNCH_4.PAC & 875.3 & 54443 & 62 & Pregnant women \\
\hline Management of maternal sepsis & MNCH_18. MMS & 928.0 & 99984 & 108 & Pregnant women \\
\hline \multicolumn{6}{|l|}{ Between $\$ 1000$ and $<\$ 10000 /$ HLY gained } \\
\hline Antibiotics for preterm premature rupture of membranes & MNCH_16. PPROM & 1863.3 & 73422 & 39 & Pregnant women \\
\hline Calcium supplementation in pregnant women for the prevention and management of pre-eclampsia/eclampsia & MNCH_5.CS & 8353.4 & 364785 & 44 & Pregnant women \\
\hline Ectopic pregnancy case management & MNCH_13.ECT & 9834.5 & 62928 & 6 & Pregnant women \\
\hline
\end{tabular}

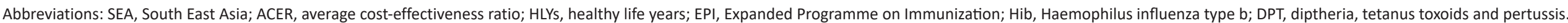


ACERs below I\$100, whereas 21 interventions demonstrate ACERs between I\$100-I\$1000 and 3 interventions fall above I\$ 1000 .

Generally, the best performing interventions are consistent across the two regions, and include:

- Family planning

- Neonatal resuscitation

- Management of pneumonia

- Vitamin A supplementation

- Management of neonatal infection (sepsis/pneumonia)

- Measles vaccine

Across both regions, ACERs below I $\$ 100$ can be found across all delivery platforms, from community to hospital level. It should be noted that all interventions classified here as "community" have ACERs below I $\$ 100$.

A comparison across countries and programme areas reveals that, out of the interventions analysed, child health and immunization produce the most favourable ACERs. Across the life course, interventions targeting the newborn have the lowest ACERs, closely followed by interventions targeting under-fives (Table 5). In terms of single interventions across the life course, the 9 newborn health interventions are among the most cost-effective, with ACERs ranging from 1.0 to 154.9 across the 2 regions (median $=14.4$ ). Next, child interventions ACERs are estimated to range between 2.5 and 581.5 (median $=15.4$ ). Finally, interventions delivered during pregnancy and child birth have ACERs which range from 0.3 to 9834.5 (median $=27.4$ ). Two interventions come out as the least costeffective across the two regions: calcium supplementation in pregnant women, and ectopic pregnancy case management.

Overall, the combination of interventions into packages produces favorable ACERs. An example is antenatal care (P2) where the package fares better than individual components such as hypertensive disease case management in pregnancy.
The reason for this is the modelled economies of scale introduced in combining facility visits and programme costs.

The design of an expansion path for SEA is illustrated in Figure 1. The first intervention is community based management of pneumonia, with an ACER of 5.0; at a cost of 49649 and 9890 HLYs gained. The second intervention included is Case management of severe neonatal infection (sepsis/pneumonia) with full supportive care. Adding subsequent interventions pushes costs upwards until the budget constraint of $\$ 4$ million is reached. Under these constraints, a total of 11 interventions and packages would be included, if cost-effectiveness was the main criteria. Most interventions included target newborn and child health outcomes.

When a 3\% discount rate was applied to benefits, ACERs were significantly higher, indicating that each HLY now came at a higher cost (Supplementary file 3). Interventions and packages that include family planning were pushed a few steps down the ranks, since the effects appear further down the time horizon Still, they remain important interventions, but now somewhat less dominant in the rank order. Aside from this effect, the rank ordering of interventions did not change. Similarly, when costs for commodities and supplies were reduced or increased by $25 \%$, the rank order did not change - indicating that drug and supply inputs are not cost drivers.

A breakdown of costs can be useful to examine cost drivers. Figure 2 provides estimates of the annual economic cost of providing the 12 packages, per capita, in I\$, in the region of SEA. Specialized health work force is an important contributor to cost for packages P3-P6; less so for the other packages.

Discussion

We have presented updated WHO-CHOICE results for

Table 5. Summary Results by Programme and Life Course Approach (Interventions and Packages Evaluated at 95\% Coverage): Comparison of ACERs Across All Countries Included in Study

\begin{tabular}{|c|c|c|c|c|}
\hline & No. of Interventions & Average ACER & Lowest ACER & Highest ACER \\
\hline \multicolumn{5}{|l|}{ SSA-E } \\
\hline Maternal and newborn health (programme) & 26 & 100.5 & 0.3 & 1156.2 \\
\hline Child health (programme) & 6 & 27.9 & 2.5 & 112.7 \\
\hline Immunization (programme) & 8 & 32.4 & 10.1 & 111.9 \\
\hline Nutrition (programme) & 9 & 211.8 & 7.1 & 1310.6 \\
\hline Pregnant women/women of reproductive age ( life course) & 22 & 194.1 & 0.3 & 1310.6 \\
\hline Newborn ( life course) & 9 & 19.4 & 1.0 & 62.7 \\
\hline Children aged 1-59 months (life course) & 18 & 27.7 & 2.5 & 112.7 \\
\hline \multicolumn{5}{|l|}{ SEA } \\
\hline Maternal and newborn health (programme) & 26 & 655.0 & 1.7 & 9834.5 \\
\hline Child health (programme) & 6 & 125.7 & 5.0 & 581.5 \\
\hline Immunization (programme) & 8 & 136.8 & 15.6 & 556.9 \\
\hline Nutrition (programme) & 9 & 1026.3 & 13.3 & 8353.4 \\
\hline Pregnant women/women of reproductive age ( life course) & 22 & 1164.8 & 11.2 & 9834.5 \\
\hline Newborn ( life course) & 9 & 46.4 & 1.7 & 154.9 \\
\hline Children aged 1-59 months ( life course) & 18 & 115.1 & 5.0 & 581.5 \\
\hline
\end{tabular}

Abbreviations: ACER, average cost-effectiveness ratio; SEA, South East Asia; SSA-E, Eastern sub-Saharan Africa. 


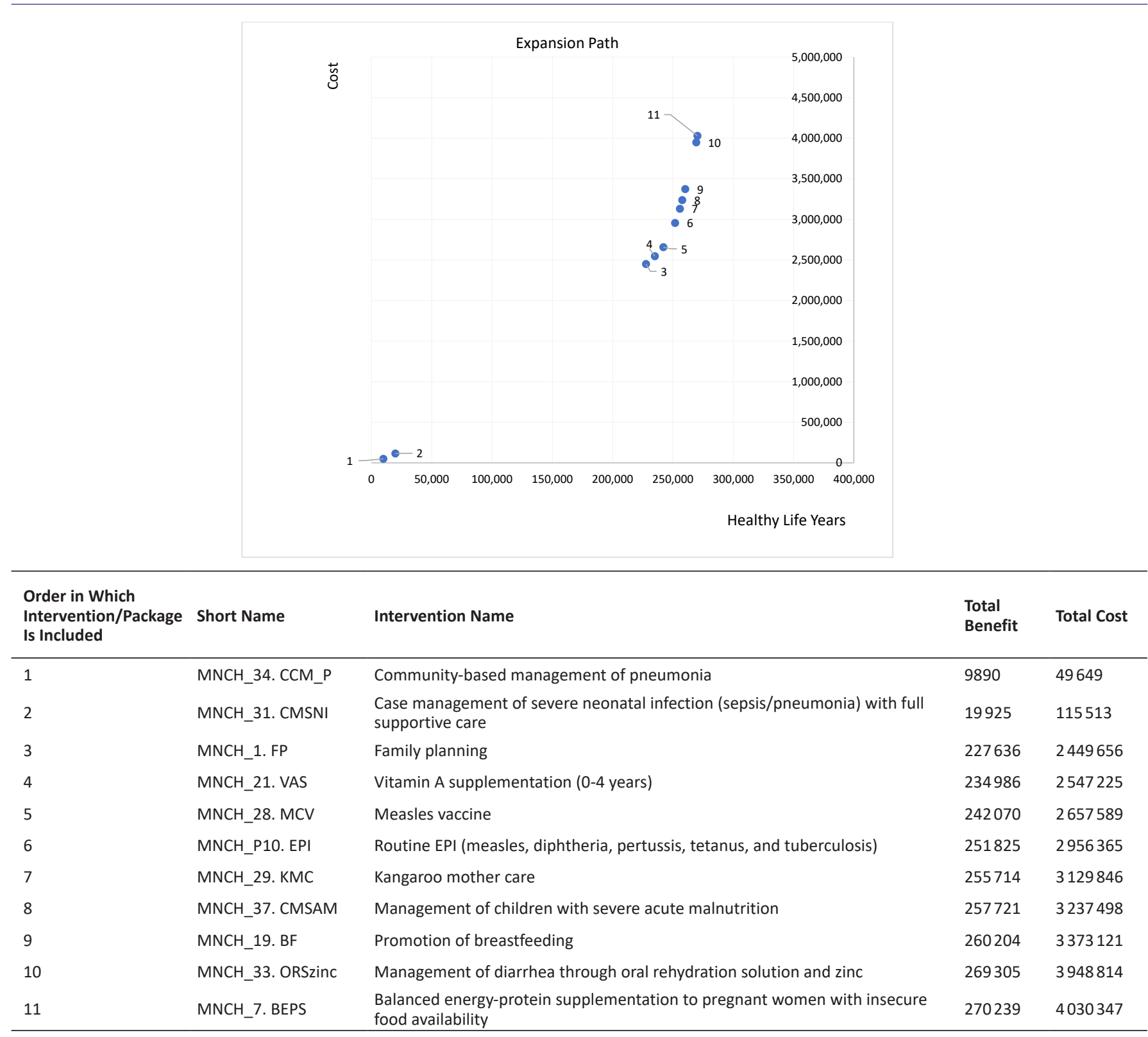

Figure 1. The Design of an Expansion Path for SEA: An Illustrative Example (Considering Interventions at 95\% Coverage). Abbreviations: SEA, South East Asia; EPI, Expanded Programme on Immunization.

interventions targeting MNCH outcomes in two geographic regions, as part of a broader update of WHO-CHOICE cost-effectiveness estimates. Examining interventions at 95\% coverage, results for SSA-E indicate that more than 39 intervention/package options are available which cost less than $\$ 100$ per healthy life year gained, with an additional 10 options under $\$ 1000$ per HLY gained (3\% discount rate for costs; $0 \%$ discount rate for outcomes). In SEA, overall costs are higher and thus ACERs are in general higher than for SSA-E. Still, 26 options cost less than $\$ 100$ per healthy life year and an additional 21 are available for ACERs less than $\$ 1000$.

Cost-effective interventions for $\mathrm{MNCH}$ can be found in all dimensions of a health system. First, we note that the I\$ 0-10 category includes interventions delivered at all platforms, from community level up to primary level and up to hospital level. It is therefore not a given that lower level service delivery platforms should be prioritized on the basis of cost-effectiveness, although other reasons may point in that direction, such as health workforce constraints. Second, cost-effective interventions exist across the life course and cover both prevention and curative interventions. Access to contraceptives through family planning stands out as an investment with high value for money. The counterfactual for the family planning intervention is a context where no-one has access to contraceptives, not even through purchase in pharmacies, which is why the model produces highly costeffective results. Third, we note the high cost-effectiveness of integrated packages across programmatic areas, including nutrition, immunization, and management of risks, such as within comprehensive antenatal care. Package options are more cost-effective than single procedures around birth (eg, management of eclampsia with magnesium-sulphate), and packages of care are also more feasible in terms of programme implementation. 


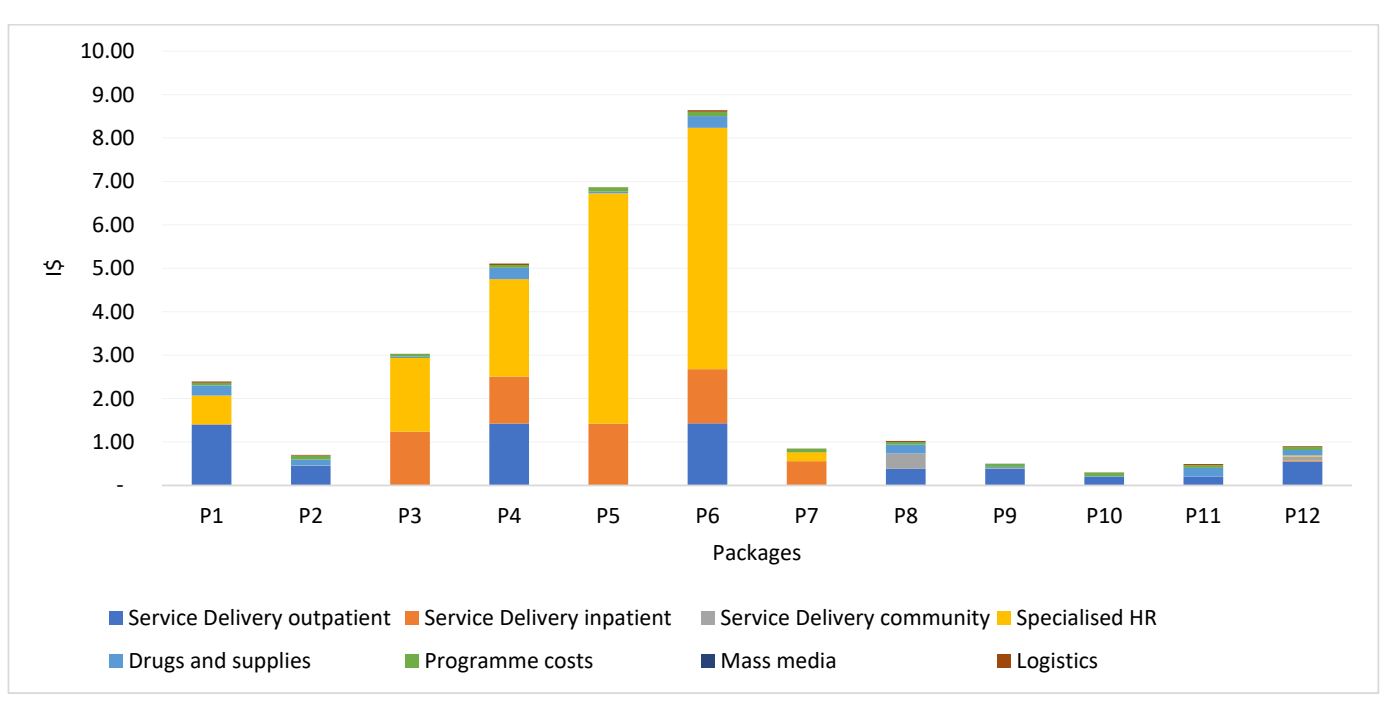

Figure 2. Annual Cost Per Capita, 12 Packages, by Cost Component (at 95\% Coverage, South East Asia region).

Similarities in rank order across the two regions are driven by the fact that both regions have high maternal and infant mortality, and that many interventions bring consistent value for money across settings - such as management of pneumonia and routine immunization. Indeed, we would not expect otherwise. However, there are important differences across regions. An example is management of maternal sepsis which is given a higher ranking in SSA than in SEA, due to the underlying burden. Across settings, there will be differences in epidemiological structure, related social and economic determinants, commodity prices, costs of health workforce and other inputs, that warrant the need for a context-specific analysis. For this analysis we have compared target coverages against a null scenario. At country level, it would be useful to also compare target coverage against current coverage, in order to assess how far off current investments are from the idealized expansion path.

Our findings are consistent with the published literature, which has previously demonstrated high cost-effectiveness of many interventions targeting MNCH outcomes. ${ }^{27}$ However, most existing publications are restricted to individual interventions, and do not compare across interventions and packages. Moreover, there is considerable variation across studies in terms of the settings/context (related to country epidemiology and delivery mechanisms), and the analytical methods used (such as time frame and discount rates). For example, many analyses do not report shared health systems costs. ${ }^{27}$ Efforts made by initiatives such as DCP3 (Disease Control Priorities, third edition) to consolidate cost-effectiveness evidence are important to the extent that they provide a landscape of the published literature, however they suffer from limitations since they compare studies that use different methods and assumptions. ${ }^{27}$ To our knowledge, the WHO-CHOICE approach is unique in generating new estimates for interventions across a range of health programmes through the use of a standardized methodological framework, which explicitly identifies and estimates shared health system costs at and above facility level.
Here we present normative estimates for specific geographic regions ("normative" referring to estimates generated for a setting with well-functioning health systems, and where best practice is followed). While there is considerable uncertainty with respect to estimates for the cost per HLY gained, the overall findings are consistent with previous analysis ${ }^{13,14}$ as we continue to find that community and facility-based newborn care, vitamin A supplementation and measles vaccine rank among the most cost-effective interventions. The most striking difference from our updated analysis is the demonstrated high value of family planning. Family planning may be regarded as a distal intervention for reducing maternal mortality as compared to clinical care during pregnancy and childbirth, however our analysis demonstrates that at population level, contraception can play an important role for mortality reduction.

Differences in intervention-specific cost-effectiveness estimates compared to the prior analysis are driven by changes in the underlying model (LiST compared to prior Excel based model) and methods (new WHO-CHOICE analysis has adopted approaches where the main scenario presented does not discount health benefits, and also lengthened the implementation period over which health benefits are modelled). While efficacy estimates have not drastically changed, the price databases used by WHO-CHOICE have been updated and costs are now estimated to be higher than in previous studies. In particular, within this analysis we have sought to specifically account for costs related to specialized health workforce, using country-specific salary estimates. ${ }^{26}$ Figure 2 demonstrated that packages that entail specific health workforce have higher costs than packages which do not require such resource. On the other hand, commodity costs are modest in comparison. This is also due to falling vaccine prices in recent years. These results underline the need to consider affordability and system constraints when prioritizing interventions for benefit packages.

The interventions analyzed conform to WHO guidelines. Our analysis shows that many interventions recommended 
by WHO are highly cost-effective, but some interventions less so. This underpins the need to consider economic analysis and resource implications within the guideline development process. An example is the WHO 2011 Calcium supplementation guideline which was revalidated in 2018, also in the context of the antenatal care. At the time, resources required for implementation were judged as high compared with other supplements such as iron and folate, and the costeffectiveness was described as "unknown."28 Here we present results that confirm the relatively high cost for implementing calcium supplementation alone, as it ranks last in both regions, though the ANC package (which included calcium supplementation) produced favorable ACERs.

\section{Limitations}

The most concerning limitation in our model is the focus on mortality outcomes, with less consideration of morbidity and overall well-being. Most interventions act on risks associated with acute events and with high mortality risks. This focus is driven by current evidence. Our analysis draws on the existing tool set for impact modelling within the Spectrum platform, which would benefit from further expansion. The LiST tool does not fully incorporate all WHO guidelines and not all relevant interventions. There are however current efforts ongoing to address these issues and expand the Spectrum platform to enable modelling a broader set of actions and outcomes, including an expanded set of essential nutrition interventions. ${ }^{29}$

Furthermore, we undertook limited uncertainty analysis. Many interventions have similar ACERs, and adjusting one or more variables could change the relative order of ranking. The expansion path presented here should therefore not be interpreted as absolute, but as an indicative example of how a country could examine the order in which to expand the coverage for different interventions.

\section{Conclusion}

Most interventions in our analysis are already being delivered in LMICs, and there is currently considerable variation in service uptake across interventions; while immunization rates are generally high, reported coverage of pneumonia treatment lags behind.

We argue that, in a context of decreasing development assistance for health, the $\mathrm{MNCH}$ agenda is still vulnerable. ${ }^{30}$ Evidence on the cost-effectiveness of interventions to improve $\mathrm{MNCH}$ outcomes must continuously be emphasized to ensure that resources are allocated to support their implementation. Beyond cost-effectiveness, criteria to consider include targeting the vulnerable, but also overall system capacity to expand coverage, and the absolute levels of investment (financing) needed for expanding service coverage. In order to enable and encourage country-level analysis that uses local data, WHO has shifted its tool set to the Spectrumbased platform which allows for such considerations. Here countries can conduct cost-effectiveness analysis using the Spectrum cost-effectiveness tool and then assess health system implications and financial costs, using the OneHealth
Tool, in both cases using the same set of impact models and applying local data and assumptions (see https://www. who.int/teams/health-systems-governance-and-financing/ economic-analysis).

It should be emphasized that, while cost-effectiveness can help identify value for money, the achievement of the SDG mortality targets requires investing in packages beyond the most easily implemented "best buys." Previous research has underlined that most MNCH-related deaths will be prevented by quality care provided at facility level. ${ }^{31}$ Reducing maternal and newborn mortality to achieve the 2030 targets will require accessible and good quality clinical services. Moreover, investments in other sectors - such as housing, agriculture, energy and education-is critical. ${ }^{32}$

With maternal and child mortality still looming high in many countries, there are opportunities to gear investments towards high-impact interventions. Evidence on cost-effectiveness can inform national processes on what to include in the benefit package from a universal health coverage perspective. These tools can be used at national level to inform the design of benefit packages, GFF investment cases and overall priority setting processes.

Ethical issues
No ethical approval was sought as this is a secondary data analysis.

Competing interests

Authors declare that they have no competing interests.

Authors' contributions

KS conceptualized the paper together with RW and MYB. KS and RW set up the models, conducted the analysis, and validated the results. All authors analyzed and interpreted the results. KS drafted the first version of the manuscript. All authors critically reviewed and edited the manuscript.

\section{Authors' affiliations}

'Department of Health Systems Governance and Financing, World Health Organization (WHO), Geneva, Switzerland. ${ }^{2}$ School of Population and Global Health, The University of Western Australia, Crawley, WA, Australia. ${ }^{3}$ Department of Nutrition for Health and Development, World Health Organization (WHO), Geneva, Switzerland. ${ }^{4}$ Department of Maternal, Newborn, Child and Adolescent Health and Ageing, World Health Organization (WHO), Geneva, Switzerland. ${ }^{5}$ Department of Sexual and Reproductive Health and Research, World Health Organization (WHO), Geneva, Switzerland. 'Department of Immunization, Vaccines and Biologicals (IVB), World Health Organization (WHO), Geneva, Switzerland.

\section{Supplementary files}

Supplementary file 1. Effect Sizes Used Within Analysis.

Supplementary file 2. Details on Cost Inputs and Prices Used in Analysis.

Supplementary file 3. Cost-Effectiveness Results by Level of Coverage, and With Varied Discount Rates.

\section{References}

1. World Health Organization (WHO). Trends in Maternal Mortality 2000 to 2017: Estimates by WHO, UNICEF, UNFPA, World Bank Group and the United Nations Population Division. Geneva: WHO; 2019.

2. World Health Organization (WHO). MDGs: Progress Made in Health. https://www.who.int/topics/millennium_development_goals/post2015/en/

3. United Nations. Transforming Our World: The 2030 Agenda for Sustainable Development. United Nations; 2015. https://sustainabledevelopment. un.org/content/documents/21252030\%20Agenda $\% 20$ for $\% 20$ Sustainable\%20Development\%20web.pdf.

4. Black RE, Victora CG, Walker SP, et al. Maternal and child undernutrition and overweight in low-income and middle-income countries. Lancet. 2013;382(9890):427-451. doi:10.1016/s0140-6736(13)60937-x 
5. Global, regional, and national disability-adjusted life-years (DALYs) for 359 diseases and injuries and healthy life expectancy (HALE) for 195 countries and territories, 1990-2017: a systematic analysis for the Global Burden of Disease Study 2017. Lancet. 2018;392(10159):1859-1922. doi:10.1016/s0140-6736(18)32335-3

6. The Partnership for Maternal, Newborn \& Child Health (PMNCH). A Global Review of the Key Interventions Related to Reproductive, Maternal, Newborn and Child Health. PMNCH; 2011.

7. Tracking Progress towards Universal Coverage for Reproductive, Newborn and Child Health: The 2017 Report. Washington, DC: United Nations Children's Fund (UNICEF), World Health Organization (WHO); 2017.

8. World Health Organization (WHO), United Nations Development Programme (UNDP). Non-Communicable Disease Prevention and Control: A Guidance Note for Investment Cases. https://apps.who. int/iris/bitstream/handle/10665/311180/WHO-NMH-NMA-19.95-eng. pdf?sequence=1. Accessed December 20, 2020.

9. World Bank Group. GFF Country Implementation Guidelines. https:// www.globalfinancingfacility.org/gff-country-implementation-guidelines. Accessed September 20, 2019. Published 2019.

10. Bertram MY, Lauer JA, Stenberg K, Edejer TT. Methods for the Economic evaluation of health care interventions for priority setting in the health system: an update from WHO-CHOICE. Int J Health Policy Manag. 2021; In Press. doi:10.34172/ijhpm.2020.244

11. Bertram MY, Chisholm D, Watts R, Waqanivalu T, Varghese C, Prasad V. Cost-effectiveness of population level and individual level interventions to combat Non-Communicable Disease in Eastern sub-Saharan Africa and South East Asia: a WHO-CHOICE analysis. Int J Health Policy Manag. 2021; Forthcoming.

12. Ralaidovy AH, Lauer JA, Pretorius C, Briët OJ, Patouillard E. Priority setting in HIV, tuberculosis, and malaria-new cost-effectiveness results from WHO-CHOICE. Int J Health Policy Manag. 2021; In Press. doi:10.34172/ijhpm.2020.251

13. Bertram MY, Lauer JA, Stenberg K, Ralaidovy AH, Edejer TT. Progressive realisation of universal health coverage in low- and middle-income countries: beyond. Int J Health Policy Manag. 2021; Forthcoming.

14. Adam T, Lim SS, Mehta S, et al. Cost effectiveness analysis of strategies for maternal and neonatal health in developing countries. BMJ. 2005;331(7525):1107. doi:10.1136/bmj.331.7525.1107

15. Edejer TT, Aikins M, Black R, Wolfson L, Hutubessy R, Evans DB. Cost effectiveness analysis of strategies for child health in developing countries. BMJ. 2005;331(7526):1177. doi:10.1136/bmj.38652.550278.7C

16. World Health Organization (WHO). WHO Recommendations on Antenatal Care for a Positive Pregnancy Experience. Geneva: WHO; 2016.

17. World Health Organization (WHO). WHO Guidelines on Maternal, Newborn, Child and Adolescent Health. WHO; 2020. https://www.who.int/ maternal_child_adolescent/guidelines/en/. Accessed February 1, 2020.

18. Walker N, Tam Y, Friberg IK. Overview of the Lives Saved Tool (LiST). BMC Public Health. 2013;13(Suppl 3):S1. doi:10.1186/1471-2458-13- s3-s

19. USAID. FamPlan - A Computer Program for Projecting Family Planning Requirements. https://lse.rl.talis.com/items/33154920-B849-F215-1A20E8C4D1B39D2B.html. Accessed October 22, 2019.

20. Index Mundi: OECD members - Contraceptive prevalence website. https:// www.indexmundi.com/facts/oecd-members/contraceptive-prevalence. Accessed February 1, 2020.

21. Stover J, McKinnon R, Winfrey B. Spectrum: a model platform for linking maternal and child survival interventions with AIDS, family planning and demographic projections. Int J Epidemiol. 2010;39(Suppl 1):i7-10. doi:10.1093/ije/dyq016

22. Salomon JA, Vos T, Hogan DR, et al. Common values in assessing health outcomes from disease and injury: disability weights measurement study for the Global Burden of Disease Study 2010. Lancet. 2012;380(9859):21292143. doi:10.1016/s0140-6736(12)61680-8

23. Avenir Health. OneHealth Tool. https://www.avenirhealth.org/software onehealth.php.

24. Bertram MY, Stenberg K, Brindley C, et al. Disease control programme support costs: an update of WHO-CHOICE methodology, price databases and quantity assumptions. Cost Eff Resour Alloc. 2017;15:21. doi:10.1186/ s12962-017-0083-6

25. Stenberg K, Lauer JA, Gkountouras G, Fitzpatrick C, Stanciole A. Econometric estimation of WHO-CHOICE country-specific costs for inpatient and outpatient health service delivery. Cost Eff Resour Alloc. 2018:16:11. doi:10.1186/s12962-018-0095-x

26. Serje J, Bertram MY, Brindley C, Lauer JA. Global health worker salary estimates: an econometric analysis of global earnings data. Cost Eff Resour Alloc. 2018;16:10. doi:10.1186/s12962-018-0093-z

27. Horton S, Levin C. Cost-effectiveness of interventions for reproductive, maternal, neonatal, and child health. In: Black RE, Laxminarayan R, Temmerman M, Walker N, eds. Reproductive, Maternal, Newborn, and Child Health: Disease Control Priorities, Third Edition (Volume 2). Washington, DC: The International Bank for Reconstruction and Development, The World Bank; 2016.

28. World Health Organization (WHO). WHO Recommendation: Calcium Supplementation During Pregnancy for Prevention of Pre-Eclampsia and its Complications. WHO; 2018.

29. World Health Organization (WHO). Essential Nutrition Actions: Mainstreaming Nutrition Through the Life-Course. WHO; 2019.

30. Institute for Health Metrics and Evaluation (IHME). Financing Global Health 2018: Countries and Programs in Transition. Seattle, WA: IHME, 2019.

31. Bhutta ZA, Das JK, Bahl R, et al. Can available interventions end preventable deaths in mothers, newborn babies, and stillbirths, and at what cost? Lancet. 2014;384(9940):347-370. doi:10.1016/s01406736(14)60792-3

32. Clark $\mathrm{H}$, Coll-Seck AM, Banerjee A, et al. A future for the world's children? a WHO-UNICEF-Lancet Commission. Lancet. 2020;395(10224):605-658. doi:10.1016/s0140-6736(19)32540-1 